\title{
Research Letter \\ Physics of Superpulses in Storage Ring Free-Electron Lasers
}

\author{
Vladimir N. Litvinenko \\ Collider-Accelerator Department, Brookhaven National Laboratory, Building 911, P.O. Box 5000, Upton, NY 11973, USA \\ Correspondence should be addressed to Vladimir N. Litvinenko, vl@bnl.gov
}

Received 11 November 2007; Accepted 14 January 2008

Recommended by Warren B. Mori

Contradictory to the widespread perception, storage ring free-electron lasers with substantial net gain can generate peak lasing power reaching GW levels in the so-called superpulse mode. This power level is sufficient for studies of nonlinear processes and efficient intracavity harmonic generation. This letter describes the physics of the superpulses and a phase-space refreshment in the electron beam responsible for this phenomenon.

Copyright () 2008 Vladimir N. Litvinenko. This is an open access article distributed under the Creative Commons Attribution License, which permits unrestricted use, distribution, and reproduction in any medium, provided the original work is properly cited.

All presently existing storage ring free-electron lasers (SR FELs) are oscillators with a two-mirror optical cavity and an optical klystron (OK) [1] comprising two wigglers and a buncher between them. SR FELs can operate in pulsed mode [2] with significant peak optical power. Early theoretical models [1] predicted peak intracavity optical power (discussed in this letter), $P_{\text {opt }}$, to be limited by

$$
P_{\text {opt }} \leq P_{e \text {-beam }} \cdot \sigma_{\gamma \text { ind }} / \gamma_{o}, \quad P_{e \text {-beam }}=\frac{\hat{I}_{e} \cdot E_{o}}{e}
$$

where $E_{o}=\gamma_{o} m c^{2}$ is the central energy and $\hat{I}_{e}$ is the peak electron beam current, $\sigma_{\gamma \text { ind }}$ is RMS spread of $\gamma=E / m c^{2}$ induced by FEL interactions, and $e$ is the electron charge. For a typical SR FEL with $E_{o} \sim 1 \mathrm{GeV}, \hat{I}_{e} \sim 10 \mathrm{~A}$, and $\sigma_{\gamma \text { ind }} / \gamma_{o} \sim 10^{-3}$, (1) limits peak FEL power to about ten MW. Studies of selfconsistent SR FEL dynamics using the 3D uvfel code [35] revealed a mechanism of superpulses with peak power two-to-three orders of magnitude exceeding the estimate (1). Figure 1 shows the typical evolution of a superpulse simulated by the uvfel code $[3,4]$, which is known for reliably predicting the electron and the optical beam evolution in SR FELs [2, 7-9].

In this letter, we discuss phenomena responsible for superpulses in SR FEL. We present a self-consistent set of dimensionless nonlinear differential equation describing superpulse and the peak power dependencies on key parameters. We also discuss the physics of superpulses using results of computer simulations for illustration ( $\sim 1000$ runs of $u v f e l$ code with $\sim 10^{6}$ macroparticles and total number of macroparticle passes through the FEL $\sim 10^{11}$ are used).
An ultrarelativistic electron is described in $6 \mathrm{D}$ phase space by its energy $E_{e}=E_{o}(1+\varepsilon)$, longitudinal coordinate $\xi=$ $v_{e} t-z$, transverse coordinates and angles $X^{T} \equiv\left[x, x^{\prime}, y, y^{\prime}\right]$, where $x^{\prime} \equiv d x / d z$ and $z$ is the coordinate along the FEL axis. Without lasing, the electron beam obtains a natural distribution in $6 \mathrm{D}$ phase space, which is close to a Gaussian and is the product of the normalized transverse $(t)$ and longitudinal $(s)$ distribution functions $f_{6 D} \cong N_{e} \cdot f_{t}(X) \cdot f_{s}$, where $N_{e}$ is the number of electrons in the e-bunch [10]. This separation of variables is valid for an SR FEL located in the dispersionfree straight section and processes much shorter than that of radiation damping time, which we discussed in this letter. In the absence of FEL interactions, longitudinal motion is an oscillation [10]

$$
\delta+i \zeta=a_{s} \cdot e^{i\left(n \Omega_{s}+\varphi_{s}\right)}, \quad f_{s}(\delta, \zeta) \cong \frac{1}{2 \pi} e^{-\left(\delta^{2}+\zeta^{2}\right) / 2}
$$

where $\Omega_{s}=2 \pi Q_{s}, Q_{s}$ is the synchrotron tune, $n$ is the turn number around the ring, and $\left\{\delta=\varepsilon / \sigma_{\varepsilon o}, \zeta=\xi / \sigma_{z o}\right\}$ are dimensionless coordinates scaled by the natural e-beam energy spread $\sigma_{\varepsilon o}=m c^{2} \sigma_{\gamma o}$ and the bunch length $\sigma_{z o}$. Trajectories in $\{\delta, \zeta\}$ phase-space are simple clock-wise circular rotations (see Figure 3).

The FEL wigglers (with period $\lambda_{w}$ and magnetic field $B_{w}$ ) provide a resonant interaction between electrons and the TEM optical wave at wavelength $\lambda_{o}$ given by

$$
\lambda_{o}=\frac{\lambda_{w}}{2 \gamma_{o}^{2}}\left(1+\left\langle\vec{a}_{w}^{2}\right\rangle\right), \quad \vec{a}_{w}=\frac{e \vec{B}_{w} \lambda_{w}}{2 \pi m c^{2}} .
$$




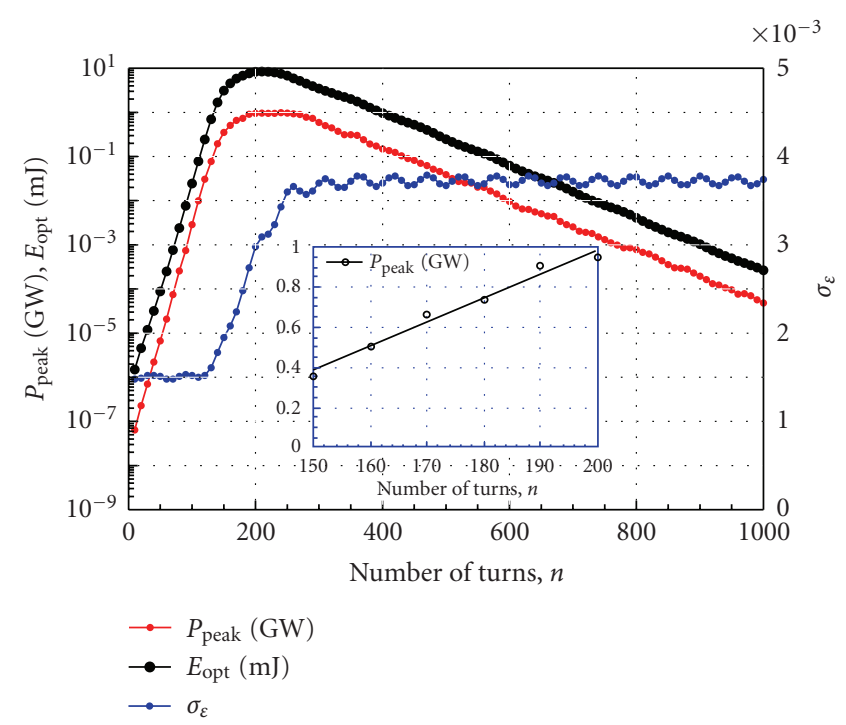

FIgURE 1: Evolution of a superpulse in OK-4/Duke SR FEL predicted by uvfel code. Intracavity optical laser beam energy (black) and peak power (red) are plotted in log-scale (left), and relative RMS e-beam energy spread (blue) is plotted in linear scale (right). Operational parameters: $E_{o}=650 \mathrm{MeV}, \hat{I}_{e}=36.3 \mathrm{~A}, \lambda=237 \mathrm{~nm}, N_{w}$ $=33.5, N_{D}=10.75, Q_{s}=0.0094, \sigma_{\gamma o} / \gamma=1.5 \cdot 10^{-3}, \sigma_{z o}=2.42 \mathrm{~cm}$, starting peak gain $G_{o}=16.5 \%$, optical cavity losses are $2 \%$ per turn (see [8] for details). Inset shows peak power growth (in linear scale) for turns 150-200.

Passing through an $\mathrm{OK}$, an electron radiates a wave packet with length equal to its total slippage $\Xi=\left(2 N_{W}+N_{D}+\right.$ $\left.O\left(\varepsilon, x^{\prime 2}, y^{\prime 2}\right)\right) \lambda_{o}, \Xi \ll \sigma_{z o}$, where $N_{w}$ is the number of periods in one wiggler and $N_{D}$ is the dimensionless slippage in the buncher [11]. When FEL interactions are present, the intracavity optical power builds up from spontaneous radiation, which is accumulated inside the optical cavity and amplified during consequent passes by the circulating e-bunch

$$
\frac{d P_{\mathrm{opt}}(\zeta)}{d n}=P_{\mathrm{opt}}(\zeta)\left(G(\zeta)-G_{\mathrm{th}}\right)+P_{\mathrm{SR}}(\zeta)
$$

where $G_{\text {th }}=\left(R_{1} R_{2}\right)^{-1}-1$ is the loss per turn in an optical cavity with mirror reflectivity $R_{1,2}$ and $P_{\mathrm{SR}}$ is the power of spontaneous radiation into the optical mode. At the beginning of the process (at $n=0 \mathrm{FEL}$ interaction is turned on $[2]$ ), the longitudinal FEL gain profile is Gaussian, like the e-beam, with peak gain $G_{0}$

$$
G_{\mathrm{FEL}}(\zeta)=G_{o} e^{-\zeta^{2} / 2}
$$

The complete set of dimensionless selfconsistent equations for a superpulse can be derived for a rather general SR FEL case, using Einstein's relations between spontaneous and induced radiation [12-14]. The optical field in an FEL can be described as a wave packet with slowly varying complex amplitude and a normalized transverse mode function $\vec{u}(\vec{r})$ :

$$
\begin{gathered}
\vec{E}_{\mathrm{opt}}=\operatorname{Re}\left[\vec{u}(\vec{r}) A_{o}(c t-z) e^{i k_{o}(c t-z)}\right], \\
\int|\vec{u}(\vec{r})|^{2} d x d y=1, \\
P_{\mathrm{opt}}(c t-z)=\frac{c}{8 \pi}\left|A_{o}(c t-z)\right|^{2}, \\
\left|\frac{d A_{o}(z)}{d z}\right| \ll \frac{\left|A_{o}\right|}{\Xi} ; \quad k_{o}=\frac{2 \pi}{\lambda_{o}} .
\end{gathered}
$$

Variation of electron energy in an FEL is the result of local interaction with a $\Xi$-long segment of the optical field and is given by an integral along the electron's trajectory

$$
\Delta E_{e}(\xi)=e \int \vec{E}_{\mathrm{opt}}\left(\vec{r}_{e}, \xi\left(\vec{r}_{e}, \varepsilon\right)\right) d \vec{r}_{e} .
$$

For a short wavelength FEL, the correlations between electrons are washed away every turn around the ring. Therefore, optical phases $\phi=k_{o}\left(c t_{i}-z_{i}\right)$ of electrons entering the FEL are random, resulting in the famous ratio between the energy loss and the energy diffusion $[12,13]$

$$
\langle\Delta \delta\rangle=\frac{d D_{\delta}}{d \delta}, \quad D_{\delta}=\frac{1}{2}\left\langle\Delta \delta^{2}\right\rangle .
$$

Using (7) and (8), one can write a set of dimensionless equations

$$
\begin{aligned}
& \frac{\partial f}{\partial \nu}+r\left(\delta \frac{\partial f}{\partial \zeta}-\zeta \frac{\partial f}{\partial \delta}\right)-p_{o}(\zeta) \cdot g \cdot \frac{\partial}{\partial \delta}\left[q(\delta) \frac{\partial f}{\partial \delta}\right]=0, \\
& \frac{d p_{o}(\zeta)}{d \nu}=p_{o}(\zeta)\left(g \int f(\zeta, \delta) \frac{-d q(\delta)}{d \delta} d \delta-1\right)+p_{\mathrm{SR}}(\zeta),
\end{aligned}
$$

with $v=n \cdot G_{\text {th }}$ as the independent variable, and optical power is normalized as $p_{0}(\zeta)=P_{\text {opt }}(\zeta) / P_{n}$ by $P_{n}=$ $\sqrt{2 \pi} \hat{P}_{e \text {-beam }} \sigma_{\gamma_{0}} / \gamma_{o}$ which is of the same order as limit in (1). Equation (9) contains dimensionless parameters $g=G_{o} / G_{\text {th }}$ (gain parameter), $r=\Omega_{s} / G_{\text {th }}$ (refreshment parameter), and a dimensionless well-defined function

$$
q(\delta)=\frac{\pi N_{e} r_{e}}{\sigma_{z o} \sigma_{\gamma o} G_{o}} \cdot \int d X^{4} f_{t}(X)\left|\int \vec{u}\left(\vec{r}_{e}\right) e^{i k_{o} \xi_{1}(X, \delta, z)} d \vec{r}_{e}\right|^{2}
$$

normalized as $\int e^{-\delta^{2} / 2}(d q(\delta) / d \delta) d \delta=-1$ to satisfy (5). Function $\xi_{1}(X, \delta, z)$ above is the electron's slippage with respect to the wave packet

$$
\xi_{1}=\xi_{10}+\int_{0}^{z}\left(\frac{1+\vec{a}_{w}^{2}(x, y, \tilde{z})}{2 \gamma_{o}^{2}}\left(1-2 \delta \sigma_{\varepsilon o}\right)+\frac{x^{\prime 2}(\tilde{z})+y^{\prime 2}(\tilde{z})}{2}\right) d \widetilde{z} .
$$




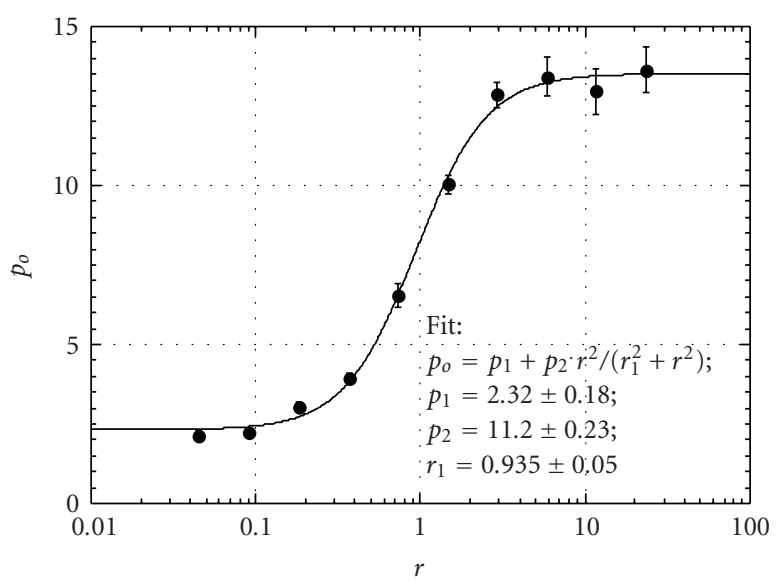

(a)

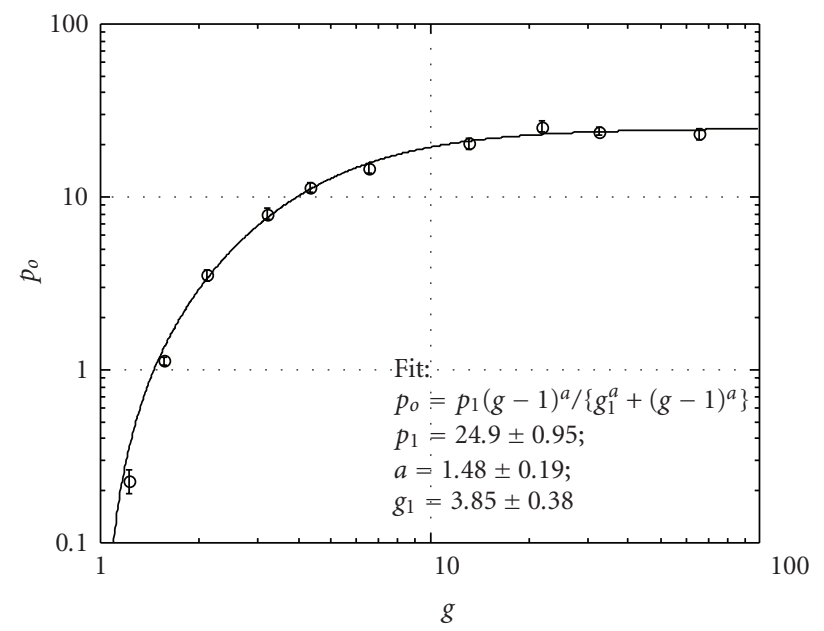

(b)

FIgURE 2: Dependencies of the normalized peak power $p_{o}$ on: (a) the refreshment parameter for $g=8.14$, (b) the gain parameter $g$ for $r=10$. Other parameters are the same as in Figure 1. Each point in the graphs shows the average value and standard error for eight to sixteen statistically independent runs of $u v f e l$ code.

For the optical beam to be amplified, the derivative of the diffusion coefficient $q(\delta)$ should be negative at and around $\delta$ $=0$. It means that $q(\delta)$ reaches its maximum at negative $\delta$ and its minimum at positive $\delta$ (see Figure 3 ). $N_{D}$ in the range

$$
1 \leq 4 \pi\left(N_{w}+N_{D}\right) \sigma_{\varepsilon o} \leq \sqrt{g}
$$

provides for attainment of maximal peak power with $|q(\delta)| \sim 1,|d q(\delta) / d \delta| \sim 1$ (the reduction of the peak power caused by finite transverse emittances of the e-beam is well described by $F_{x, y}$ factors in [8]). Figure 2 shows dependencies of peak power on the gain and the refreshment parameters. The power is low close to the lasing threshold $(g-1 \ll 1)$, grows very fast in $1.5<g<10$ range, and saturates above $g=$ 10. Dependence on the refreshment parameter is less dramatic, but peak power increases about sixfold from $r \ll 1$, when the synchrotron motion is essentially frozen, to $r \gg 1$. Detailed studies show that optimal ratio between $r$ and $g$ is near $\pi$, that is, when electrons go through half of a syn- chrotron oscillation during one e-growth time $v_{e}=1 / \mathrm{g}$ of the laser power. Enhancement comes from synchrotron oscillations moving electrons through the center region and allowing the short optical pulse to be amplified by entire electron bunch. Further increase of the refreshment parameter is not beneficial and can cause oscillations of optical power.

Figure 3 shows details of the e-beam dynamics. During the superpulse, period of accumulation of spontaneous radiation is followed by a period of exponential growth. The correlation length of the optical field grows with the amplification $\Gamma=\int G_{\mathrm{FEL}}(n) d n$ as $\xi_{\text {corr }} \cong \Xi \sqrt{1+\Gamma}$, while the envelope of the optical pulse shrinks $\sigma_{\text {opt }} \propto 1 / \sqrt{1+\Gamma}[6]$ to about $30 \%$ of the initial e-bunch length. The initial power growth by 5 to 6 orders of magnitude (see Figures 1 and 3, turns 1100) does not affect significantly the e-beam distribution and the gain profile. The amplified optical pulse grown from the "white" noise consists of very short $\left(\Delta \zeta=1-2 \cdot 10^{-3}\right)$ spikes with peak power in the spikes exceeding the local average by approximately an order of magnitude, similar to that in SASE FELs $[15,16]$.

Further growth of the optical power affects the e-beam distribution and complicates the dynamics. Energy loss and diffusion in the FEL make the phase-space distribution of electrons asymmetric and time-dependent. The growth of the local energy spread reduces the FEL gain while the asymmetric gain profile and energy diffusion cause a drift of the optical pulse and the electron beam from the center $[7,18]$ see changes in $\langle\delta\rangle,\langle\zeta\rangle$ in Figure 3. The optical power reaches maximum when net gain crosses zero at $G_{\mathrm{FEL}}=G_{\mathrm{th}}$. After that, the optical power decays, and the electron beam distribution becomes more symmetric.

As shown in "Turn 200" plot in Figure 3, lower energy electrons $(-3<\delta<0)$ have maximum diffusion, while electrons with higher energy have a low diffusion energy corridor clearly noticeable at $1<\delta<3$. This diffusion asymmetry, which is fundamental to the amplification process in FEL $[12,13]$, produces the top-bottom asymmetry in the Poincaré plots. Electrons at large amplitude of synchrotron oscillations $\left(a_{s} \geq 1\right)$ interact with the intense optical pulse briefly $\left(n_{\text {int }} \propto \sigma_{\text {opt }} / a_{s} \Omega_{s}\right)$ while passing near the $\zeta=0$. The diffusion imprints the level of the FEL power at that moment onto the distribution. Thus, the energy spread seen at angle $\psi$ in Figure 3 shows that the FEL power level happened about $\Delta n \cong \psi / \Omega_{s}$ turns in the past. Thus, the segment $0<$ $\psi<\pi$ in the turn 200 graph shows the diffusion imprinted at negative $\delta$ between turns 147 and 200. Similarly, the segment $\pi<\psi<2 \pi$ shows the diffusion imprinted at positive $\delta$ during the same turns. Thus, the snail-like dependence of the amplitude spread is a record of the evolution of the optical pulse power (see clip in Figure 1) as well as a clear indication of the diffusion asymmetry. Such snail-like distribution of electron beam was also observed experimentally [19].

The asymmetry in diffusion creates multipole moments in longitudinal phase space and residual quadrupole oscillations with frequency $2 \Omega_{s}$ can be seen in the oscillations of RMS energy spread in Figure 1.

The dimensionless nature of (8) provides for the scalability of the results presented in this letter: SR FELs with the same parameters $\{r, g, q(\delta)\}$ would have the same average 


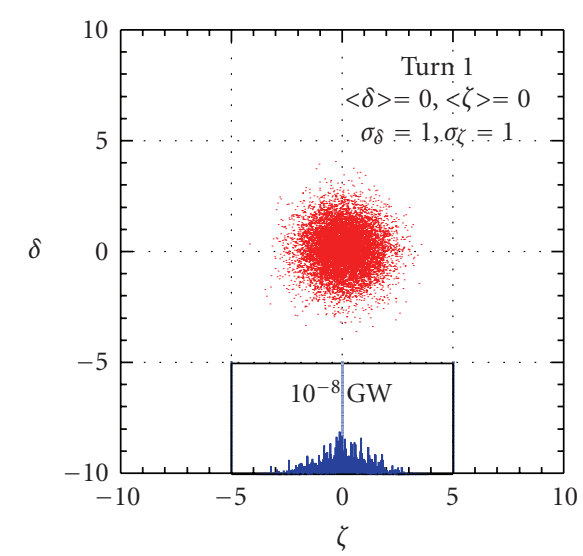

(a)

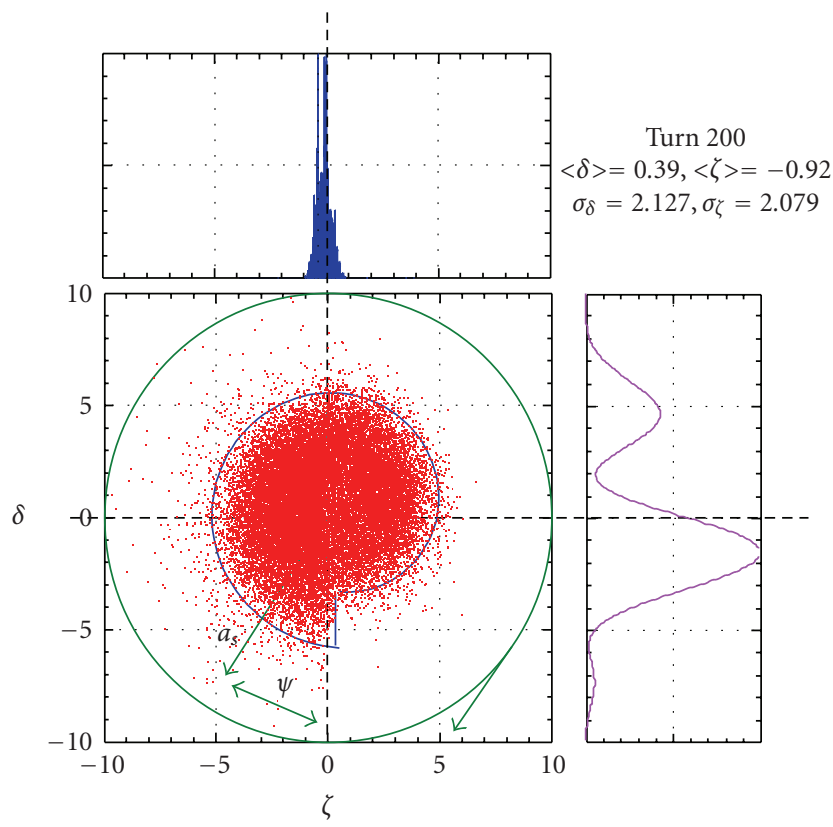

(c)

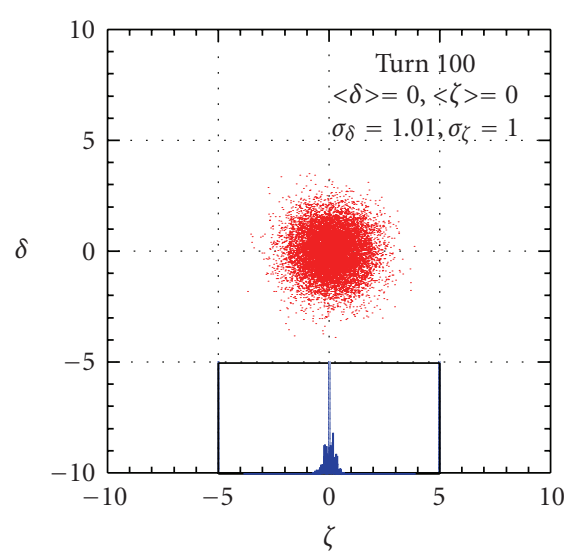

(b)

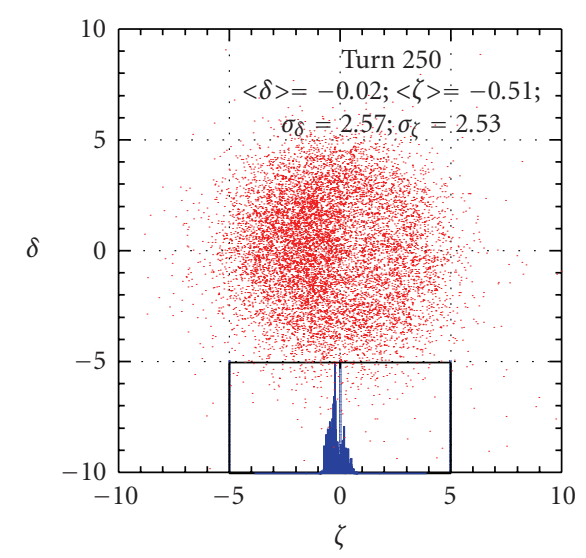

(d)

FIGURe 3: Four Poincaré plots (electrons are red dots) in $(\delta, \zeta)$ phase space during the superpulse (parameters are as in Figure 1). The clips show the $\zeta$-profiles of the optical power (blue) with power scales $10 \mathrm{~W}$ for turn $1,10 \mathrm{MW}$ for turn 100 , and $1 \mathrm{GW}$ for turns 200 and 250 . The Turn 200 plot has additional clip on the right with the FEL energy diffusion function. The RMS length of optical pulse is $\sigma_{\mathrm{opt}}=0.35$ at turn 200. The circle and arrow show the trajectory of synchrotron motion for a particle with $a_{s}=10$.

values and variations of the dimensionless peak power $p_{0}$. The peak superpulse power can be then simply obtained through multiplication by $P_{n}$. Thus, the simulation results presented in this letter can be used for predicting parameters in many SR FELs.

Overall, the phenomena of superpulses in SR FELs, which are presently verified both experimentally $[18,19]$ and confirmed by other FEL codes [20], open a range of new opportunities for generating coherent femtosecond Xray pulses in SR FELS and their scientific applications. The OK-4 FEL at Duke produced superpulses at wavelengths of 240-270 $\mathrm{nm}$ for efficient coherent harmonic generation from $130 \mathrm{~nm}$ to $37 \mathrm{~nm}[9,18]$. These experiments were followed by successful harmonic generation in Elettra SR FEL [17].
These experiments also support predictions for superpulses presented in this letter [20].

\section{REFERENCES}

[1] N. A. Vinokurov and A. N. Skrinsky, "Preprints 77-59 and 7767," Novosibirsk, Russia, 1977.

[2] I. V. Pinayev, V. N. Litvinenko, S. H. Park, et al., "Giant highpeak power pulses in the UV OK-4/Duke storage ring FEL using the gain modulator," Nuclear Instruments and Methods in Physics Research Section A, vol. 475, no. 1-3, pp. 222-228, 2001.

[3] V. N. Litvinenko, B. Burnham, J. M. J. Madey, and Y. Wu, "High peak pulse power operation of the OK-4/Duke XUV FEL," in Time-Resolved Electron and X-Ray Diffraction, 
vol. 2521 of Proceedings of SPIE, pp. 78-89, San Diego, Calif, USA, July 1995.

[4] V. N. Litvinenko, B. Burnham, J. M. J. Madey, and Y. Wu, "Giant laser pulses in the Duke storage ring UV FEL," Nuclear Instruments and Methods in Physics Research Section A, vol. 358, no. 1-3, pp. 334-369, 1995.

[5] V. N. Litvinenko, B. Burnham, J. M. J. Madey, and Y. Wu, "Dynamics of the Duke storage ring UV FEL," Nuclear Instruments and Methods in Physics Research Section A, vol. 358, no. 1-3, pp. 334-369, 1995.

[6] V. N. Litvinenko and N. A. Vinokurov, "Lasing spectrum and temporal structure in storage ring FELs: theory and experiment," Nuclear Instruments and Methods in Physics Research Section A, vol. 304, no. 1-3, pp. 66-71, 1991.

[7] V. N. Litvinenko, S. H. Park, I. V. Pinayev, and Y. Wu, "Time structure of the OK-4/Duke storage ring FEL," Nuclear Instruments and Methods in Physics Research Section A, vol. 475, no. 1-3, pp. 240-246, 2001.

[8] V. N. Litvinenko, S. H. Park, I. V. Pinayev, and Y. Wu, "Power limitations in the OK-4/Duke storage ring FEL," Nuclear Instruments and Methods in Physics Research Section A, vol. 475, no. 1-3, pp. 65-73, 2001.

[9] V. N. Litvinenko, "New results and prospects for harmonic generation in storage ring FELs," Nuclear Instruments and Methods in Physics Research Section A, vol. 507, no. 1-2, pp. 265-273, 2003.

[10] H. Wiedemann, Particle Accelerator Physics, Springer, Berlin, Germany, 1993.

[11] P. Elleaume, "Storage ring FEL theory," Nuclear Instruments and Methods in Physics Research Section A, vol. 237, no. 1-2, pp. 28-37, 1985.

[12] V. N. Litvinenko and N. A. Vinokurov, "On the classical analog of the Einstein relations between spontaneous emission, induced emission and absorption," Nuclear Instruments and Methods in Physics Research Section A, vol. 331, no. 1-3, pp. 440-449, 1993.

[13] J. M. J. Madey, "Relationship between mean radiated energy, mean squared radiated energy and spontaneous power spectrum in a power series expansion of the equations of motion in a free-electron laser," Il Nuovo Cimento B, vol. 50, no. 1, pp. 64-88, 1979.

[14] A. Einstein, Papers, Four Volumes, vol. 3, Nauka, Moscow, Russia, 1966.

[15] E. L. Saldin, E. A. Schneidmiller, and M. V. Yurkov, The Physics of Free-Electron Lasers, Springer, Berlin, Germany, 2000.

[16] C. Pellegrini, "Design considerations for a SASE X-ray FEL," Nuclear Instruments and Methods in Physics Research Section $A$, vol. 475, no. 1-3, pp. 1-12, 2001.

[17] G. De Ninno, "Coherent harmonic generation using the ELETTRA optical klystron," in Proceedings of the 26th International Free Electron Laser Conference (FEL '04), pp. 237-240, Trieste, Italy, August 2004.

[18] E. Longhi, Coherent harmonic generation in storage ring free electron lasers, Ph.D. thesis, Duke University, Durham, NC, USA, 2006.

[19] K. Chalut, V. N. Litvinenko, and I. V. Pinayev, "Method of phase-space tomography of rapidly evolving e beams," Physical Review Special Topics-Accelerators and Beams, vol. 8, Article ID 102802, 7 pages, 2005.

[20] G. De Ninno, et al., "Self-induced harmonic generation in a SR FEL," accepted for publication in Physical Review Letters on January 18, 2008. 

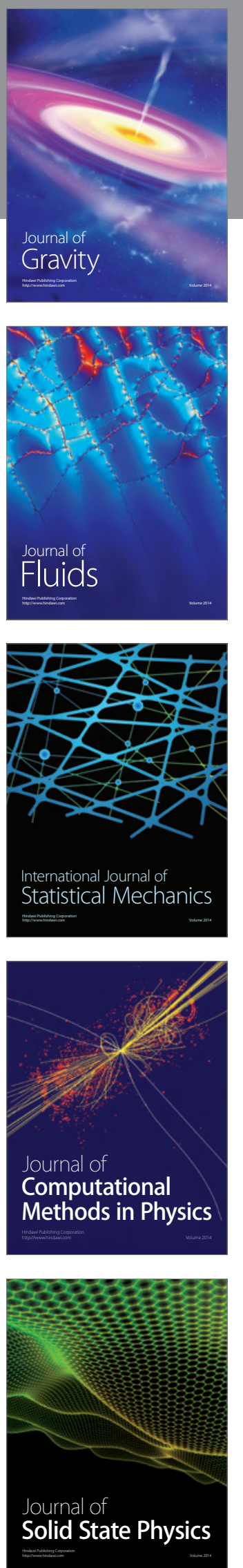

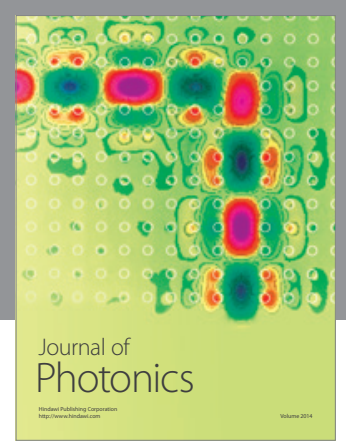

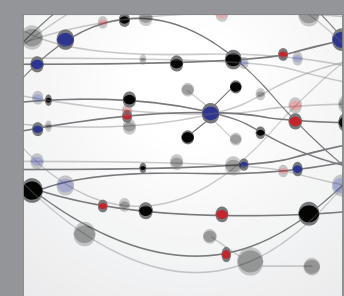

The Scientific World Journal
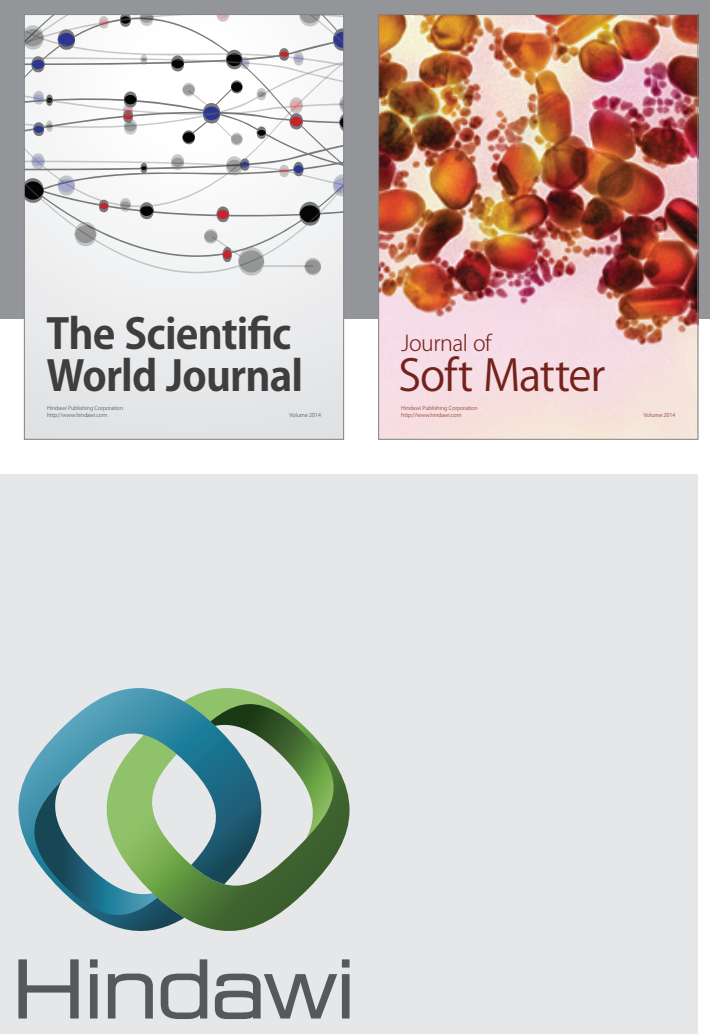

Submit your manuscripts at

http://www.hindawi.com
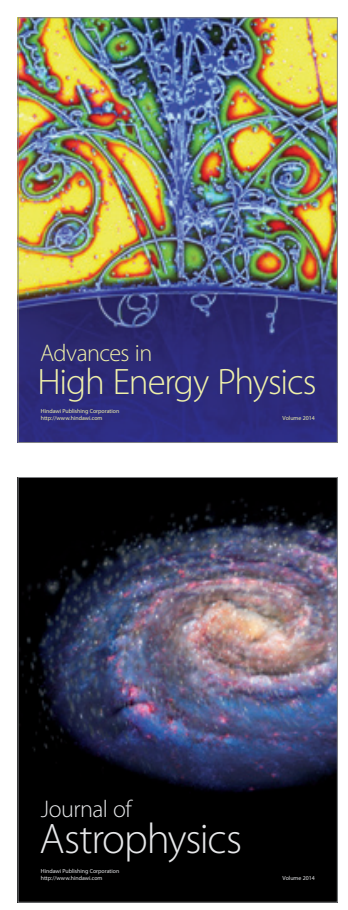
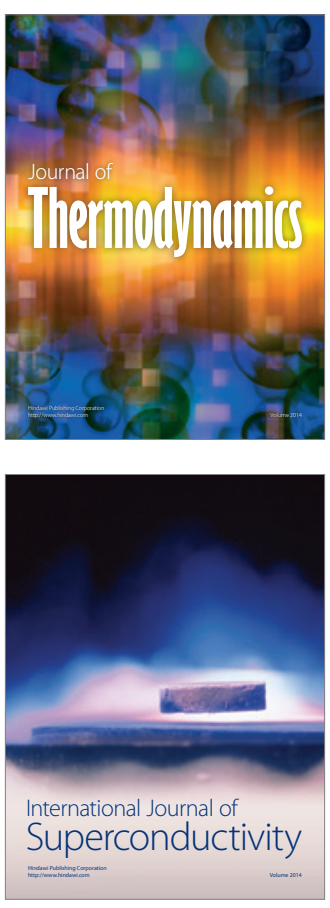
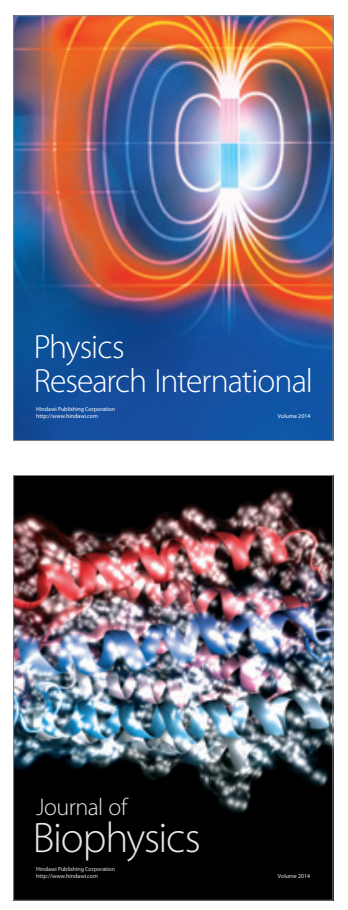
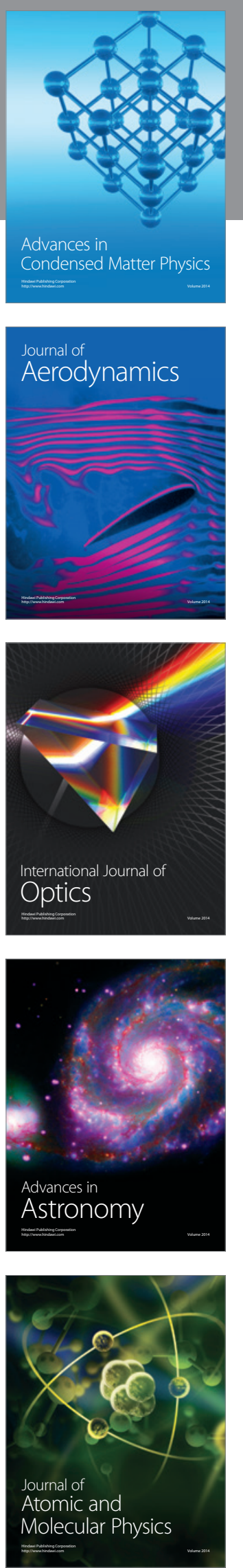